\title{
Former des étudiants LEA à la traduction technique et scientifique : un défi didactique?
}

\section{Élisabeth Lavault-Olléon}

\section{(2) OpenEdition}

\section{Journals}

Édition électronique

URL : https://journals.openedition.org/asp/4238

DOI : 10.4000/asp.4238

ISSN : 2108-6354

Éditeur

Groupe d'étude et de recherche en anglais de spécialité

Édition imprimée

Date de publication : 1 février 1994

Pagination : 65-82

ISSN : 1246-8185

Référence électronique

Élisabeth Lavault-Olléon, « Former des étudiants LEA à la traduction technique et scientifique : un défi didactique? », ASp [En ligne], 3 | 1994, mis en ligne le 20 février 2014, consulté le 21 septembre 2021. URL : http://journals.openedition.org/asp/4238 ; DOI : https://doi.org/10.4000/asp.4238

Ce document a été généré automatiquement le 21 septembre 2021.

Tous droits réservés 


\title{
Former des étudiants LEA à la traduction technique et scientifique : un défi didactique?
}

\author{
Élisabeth Lavault-Olléon
}

\section{Cadre et fondements théoriques}

\subsection{Présentation}

Pour situer mon intervention, je rappellerai que j'enseigne depuis trois ans à l'Université Stendhal, au département LEA, et que je m'y occupe principalement de traduction, en particulier de traduction spécialisée. Notre deuxième cycle LEA comprend, en effet, une filière de traduction spécialisée qui débouche sur un DESS intitulé "Traduction spécialisée et production de textes multilingues » que nous avons ouvert cette année et dont je suis responsable. J'ai moi-même enseigné plusieurs années à l'ESIT comme chargée de cours, et c'est dans l'équipe de chercheurs de l'ESIT que j'ai effectué mes recherches, notamment ma thèse sur les «Fonctions de la traduction en didactique des Langues».

\subsection{Opposition traduction pédagogique/traduction professionnelle}

2 Je ne parlerai ici ni de thème ni de version, qui sont des exercices académiques consacrés à l'enseignement des langues. Je ne les critiquerai pas non plus - ils ont leur valeur, toute relative, dans l'enseignement du vocabulaire, de la grammaire et du style, tant en français que dans la langue apprise - mais ils n'apprennent pas à traduire. Je me place dans l'optique d'un enseignement de la traduction professionnelle qui ne concerne pas la transposition d'un texte $\mathrm{A}$ en un texte $\mathrm{B}$, mais qui met l'étudiant dans la situation suivante : un auteur - un chercheur, un constructeur, un fabricant - a produit un texte en langue source pour un public donné, lecteur de la langue source. Ce texte contient un 
certain nombre d'informations que le public analogue, lecteur de la langue cible, désire connaître. Le traducteur est chargé, soit par ce public, soit par l'auteur, de transmettre ces informations dans la langue cible afin que le destinataire final, lecteur de la langue cible, les comprenne. Il s'agit là d'un acte de communication qui n'a rien à voir avec l'exercice de version dans lequel la communication est didactique, entre professeur et élève, et concerne les mécanismes des deux langues, mais où il n'y a pas de transmission de l'information contenue dans le texte puisque le commanditaire de la traduction (le professeur) la connaît. Mon approche de la traduction n'est donc pas linguistique, elle est résolument communicative.

\subsection{Traduction de textes pragmatiques et théorie du sens}

3 L'approche qui convient à ce type de traduction s'appuie sur la théorie du sens ou théorie interprétative de la traduction, développée par Danica Seleskovitch à l'ESIT (1984), et que j'ai déjà abondamment commentée dans d'autres lieux. Je rappellerai qu'elle redonne au traducteur sa place centrale en tant que moteur interprétant le texte source et le réexprimant en langue cible, et qu'elle décompose le processus de traduction en trois phases: la première phase, l'interprétation du texte, se doit de prendre en compte beaucoup plus que l'expression linguistique du texte, à savoir le contexte verbal et le contexte situationnel, c'est-à-dire toutes les données de la situation d'énonciation du texte source. Parmi celles-ci, l'analyse du vouloir-dire (ou intention de signifier) de l'auteur (avec ses implicites) et le ciblage du destinataire de la traduction sont des éléments déterminants. L'interprétation du sens doit aboutir à une deuxième phase, la déverbalisation du texte source : le traducteur «oublie» les mots du texte source pour n'en retenir que le sens, et pouvoir aborder enfin la troisième phase, la restitution dans la langue cible, sans contamination de la langue source.

4 Cette décomposition quelque peu abstraite du processus de traduction a des applications évidentes en didactique de la traduction qui me semblent le B A BA de tout cours de traduction :

- l'analyse du texte à traduire, qui implique la connaissance de toutes les données paralinguistiques du texte: d'où un travail sur des textes intégraux au contexte connu et l'analyse préalable des composantes de la communication (Delisle 1980),

- l'entraînement à la déverbalisation par des exercices spécifiques,

- l'entraînement à la rédaction (respect des contraintes de la langue cible, adéquation des styles, élimination des calques, adaptation au destinataire).

Dans un cours de traduction générale portant sur la traduction de textes pragmatiques, essentiellement tirés de la presse courante, cette approche est une première étape vers l'apprentissage de la traduction.

6 Mon propos d'aujourd'hui concerne la formation à la traduction spécialisée proprement dite, qui s'effectue non pas dans le cadre du cours général de traduction présent dans toutes les UV de langues en LEA, mais dans le cursus de traduction spécialisée qui commence en licence, se poursuit en maitrise et aboutit au DESS.

7 La traduction professionnelle se caractérise par la prise en compte de la situation professionnelle (travail commandité, établissement d'un contrat, respect des contraintes imposées par le client, respect de délais généralement très courts, etc.) mais aussi par un 
type de textes qui n'est pas habituellement présent en cours de traduction, le texte spécialisé ou le texte technique.

\section{La traduction technique ou spécialisée en LEA}

\subsection{Types de textes}

Puisqu'il s'agit d'une formation professionnelle, l'étude du marché est à la base du choix de textes traduits. Il est certain que les extraits de Time ou de The Economist du cours de traduction générale ne font jamais l'objet de demandes de traduction professionnelle. Les textes demandés sont de deux sortes :

\section{Documents techniques ou technico-commerciaux}

Notes techniques, manuels d'utilisateurs, notices d'assemblage, modes d'emploi, brochures de présentation des produits, appels d'offres, contrats.

Ces textes sont des textes opératoires, c'est-à-dire qu'ils ne transmettent pas simplement des informations, ils conduisent à l'exécution d'une tâche, ou à une réalisation qui, si elle est réussie, est la garantie de la réussite de la rédaction, et donc de la traduction.

\section{Veille technologique et articles spécialisés}

11 Ce sont des textes informatifs dans des domaines économiques, scientifiques et techniques de pointe. Ils se situent généralement en amont des textes opératoires de la première catégorie.

12 Je parlerai dans les deux cas de traduction spécialisée. Précisons aussi que la traduction s'effectue vers le français pour être conforme aux exigences de qualité de la profession, qui implique toujours une traduction rédigée dans la langue maternelle du traducteur.

\subsection{Spécificités pour le public LEA}

\subsubsection{Caractéristiques du texte spécialisé}

Analysons-le de façon globale, en le comparant au texte de presse auquel les étudiants sont habitués. Il se distingue par :

- sa longueur qui peut varier d'une page à 300 pages, en tout cas toujours plus que les 30 lignes de la version standard,

- son aspect discontinu, morcelé en instructions, schémas, illustrations et leurs légendes, listes, etc.

- un contenu souvent non compréhensible à première lecture du fait de :

- la densité élevée de vocabulaire spécialisé, y compris de chiffres, de formules, et d'unités de mesure,

- l'opacité du sujet due à l'ignorance du domaine et des concepts de base.

\subsubsection{Les étudiants en LEA}

Soumettez un texte spécialisé standard - un manuel d'utilisateur d'un logiciel de 5 pages, ou une notice d'assemblage d'un appareil électrique - aux étudiants LEA de licence ou de maîtrise et vous créez une telle panique qu'une majorité d'entre eux fuit irrémédiablement vers les cieux plus cléments et plus familiers de l'économie et de la gestion dans l'option Affaires et Commerce. 
Les étudiants en LEA sont dans leur grande majorité des étudiants titulaires du bac A2 Lettres et Langues : sans être réellement littéraires, ils ne sont pas formés aux sciences et techniques. Leur orientation au lycée puis à l'université s'est d'ailleurs fondée davantage sur un rejet des mathématiques et de la technique que sur une réelle passion pour les langues. Le blocage est donc ancré, et bien souvent irréversible.

Pourtant, après avoir compris ce qu'on attendait d'eux dans la filière "Traduction spécialisée ", ils sont un petit nombre à vouloir poursuivre la formation. Il est vrai que parmi ceux-ci se trouvent justement les quelques étudiants atypiques titulaires d'un bac scientifique ou technique. Mais il y a aussi des étudiants de profil A2, et ce sont surtout ceux-là qui méritent toute notre attention didactique et une progression adaptée.

\section{Quatre étapes dans une progression d'apprentissage}

\subsection{Première étape : texte opératoire de base}

18 Voici une recette de cuisine américaine, tirée d'un livre de cuisine végétarienne qui n'a jamais été traduit. Et quel dommage! Ses recettes sont si délicieuses qu'il serait très agréable d'en goûter une, préparée par votre tante ou votre grand-mère qui aime tant cuisiner mais qui ne connaît pas l'anglais. Alors traduisez-la pour elle. Attention : c'est une Française moyenne, qui ne connaît rien des produits et mesures spécifiquement américains, qui fait ses courses au supermarché du quartier et possède une balance et un four standards.

Alors au travail !

20

Et voici ce que j'obtiens.

\subsubsection{Exemple 1}

Tarte Cosaque

Temps de préparation : deux heures environ

Préchauffer le four à $350^{\circ}$

Recette pour 6 personnes (moule à tarte de $23 \mathrm{~cm}$ )

Ingrédients :

1 fond de tarte de $23 \mathrm{~cm}$

$125 \mathrm{~g}$ de champignons frais

$200 \mathrm{~g}$ d'oignons hachés

Mettre au four pendant 40 minutes à $350^{\circ}$

\subsubsection{Commentaire}

Inutile de corriger la recette : de toute façon, la tarte est brûlée.

22

Diagnostic : l'étudiant a oublié de convertir les degrés Fahrenheit en Celsius. NB : il n'y avait effectivement ni $\mathrm{F}$ ni $\mathrm{C}$ à côté de $350^{\circ}$. Mais il ne s'agit pas ici de transposer des mots et des chiffres sans réfléchir, il s'agit de traduire le message d'une personne pour une autre, et de prendre en compte la situation de communication de départ: une recette rédigée par une Américaine pour des Américains, où le $\mathrm{F}$ de Fahrenheit est implicite. La 
conversion des pouces en centimètres a, elle, été correctement réalisée, car l'unité de mesure était explicite.

\subsubsection{Exemple 2}

Ingrédients :

1 fond de tarte de $23 \mathrm{~cm}$

$113 \mathrm{~g}$ de champignons frais

1 tasse d'oignons hachés

1 tasse de chou vert râpé

1 tasse de brocolis émincés

1 tasse de carottes émincées

1 échalote hachée menu

Le tiers d'une tasse de fromage blanc

$3 / 4$ de tasse de yaourt et de crème fraîche mélangés

\subsubsection{Commentaire}

Diagnostic :

Perplexité de la grand-mère : pourquoi diable $113 \mathrm{~g}$ de champignons et non pas $120 \mathrm{~g}$ ou $125 \mathrm{~g}$, soit $1 / 4$ de livre ? Et quelle tasse choisir ? Une tasse à café, à thé, à chocolat? Leur contenance varie du simple au double.

Résultat : Pas de tarte. La recette semble bizarre et la grand-mère n'a pas du tout envie de se compliquer la vie.

L'étudiant a converti $1 / 4$ de livre américaine en grammes, sans penser à l'utilisateur qui a une balance graduée tous les 10 grammes et qui est habitué aux chiffres ronds. L'arrondissement des chiffres est une constante de tous les guides d'utilisation. Pour être opératoires, les chiffres et les mesures doivent entrer dans une catégorie reconnue, s'apparenter à un modèle générique : la ménagère accepte $125 \mathrm{~g}$ de champignons ou $500 \mathrm{~g}$ de carottes, tout en sachant pertinemment qu'elle prendra peut-être $32 \mathrm{~g}$ ou $50 \mathrm{~g}$ de plus ou de moins. Mais $116 \mathrm{~g}$ de champignons, ou $501 \mathrm{~g}$ de pommes de terre nous font basculer dans l'inconnu, parce que non conformes au code. C'est plus que gênant, c'est rebutant pour l'utilisateur.

L'erreur provient de la méconnaissance du code implicite de rédaction technique, et du manque d'adaptation au destinataire de la traduction (et à sa balance).

L'étudiant a également oublié de vérifier qu'une «cup » américaine est bien une unité de mesure de capacité qui correspond à 8 fluid ounces, soit $225 \mathrm{ml}$, un peu moins d'un quart de litre - un peu plus qu'une tasse à chocolat, un peu moins qu'un bol. De toute façon, les Français n'ont pas l'habitude de mesurer du chou ou des carottes avec une tasse, ils les pèsent. Il faut donc convertir ces volumes en grammes, avec une variation selon la densité des aliments - mais qui va peser du chou ou des brocolis à 10 grammes près ? Là aussi il convient d'arrondir en disant « entre 150 et $200 \mathrm{~g}$ ».

Même chose pour le fromage blanc et la crème, sauf qu'en France, on vend le premier en pots de $125 \mathrm{~g}, 250 \mathrm{~g}$ ou $500 \mathrm{~g}$ et la seconde en pots de $10 \mathrm{cl}, 20 \mathrm{cl}, 50 \mathrm{cl}$. L'adaptation se fait en fonction de ce qui est offert dans les magasins.

La conversion exacte donne 7,5 $\mathrm{cl}$ de fromage blanc, soit $10 \mathrm{cl}$ ou $70 \mathrm{~g}$ en arrondissant, mais la grand-mère préférera à coup sûr lire « trois cuillerées à soupe de fromage blanc ». 
30 Là aussi, le traducteur doit mesurer l'impact que produira son texte sur le destinataire et l'adapter pour qu'il soit reçu de manière optimale.

\subsubsection{Autres exemples}

31 À ces deux exemples, qui témoignent d'une part d'une erreur d'interprétation du texte source et d'autre part d'un manque d'adaptation au public cible, je pourrais en ajouter beaucoup d'autres, dus à la méconnaissance de l'anglais :

- "ground caraway seed» traduit par «graines de cumin» (oubli de «moulu» ou «en poudre ») ou «fresh black pepper» par " poivre noir en grains » : dans les deux cas, la tarte, ainsi agrémentée de graines de cumin et de grains de poivre perd beaucoup de son charme.

- " add egg-cheese purée to sautéed vegetables and mix well » a été traduit par « ajouter le mélange œufs-fromage aux légumes et mixer le tout ». Pourquoi avoir passé une demi-heure à couper les légumes en petits morceaux, si c'est pour les passer au mixeur?

\subsubsection{Conclusions}

Dans tous les cas, il existe un moyen concret de valider la traduction, c'est de réaliser la recette soi-même, ou de la faire réaliser par une autre personne à partir de la traduction. Le verdict est sans appel. Il fait rire les étudiants, mais il est un avant-goût du verdict du technicien qui ne peut assembler sa machine parce que la notice d'assemblage est incompréhensible - et qui mène un client à refuser de payer le traducteur...

Ce choix d'une recette de cuisine n'est pas anodin : sous ses apparences ordinaires, voire simplistes, la recette présente, à un niveau relativement simple, tous les caractères du document technique et toutes ses difficultés :

- elle décrit une "technique » qui conduit à une mise en œuvre, validation irréfutable de la traduction (réussite ou non de la recette).

- Elle possède une terminologie et une phraséologie propres (pour "mushroom stems », on parle des " pieds » des champignons et non pas des « queues »; pour " pie crust » de " pâte » et non pas de «croûte»; on ajoute « une noix de beurre » et non pas « un peu de beurre »; on va « retirer du feu » et non pas « ôter du feu»; la formule consacrée pour « remove stems from mushrooms » est " ôtez le pied sableux des champignons », etc.

- Elle est soumise à des contraintes stylistiques (phrases courtes, choix de l'impératif ou de l'infinitif, de l'article défini plutôt que du partitif, par exemple « retirer du feu et ajouter la farine et le vin » et non pas « de la farine et du vin »).

- Elle est conforme à un code de rédaction : chiffres arrondis, élimination des ambiguïtés, cohérence des termes et non modulation des répétitions. Contrairement à ce que l'on pratique en traduction journalistique, il convient de toujours employer le même terme pour le même objet: par exemple, «le mélange œufs-fromage » ne doit pas devenir à la ligne suivante « la garniture », puis « la préparation précédente », etc.).

- Elle exige un ordre car elle présente une procédure (étape 1 avant 2, etc.).

- Elle est présentée suivant un modèle relativement standardisé (temps de préparation et ustensiles en haut, listes des ingrédients avant la réalisation, etc.).

- Elle s'adresse, dans la plupart des cas, à des spécialistes, ou du moins à des habitués (d'où la totale incapacité de traduire d'un étudiant africain pour qui la cuisine est uniquement «affaire de femmes »). Cependant, puisqu'il s'agit, malgré tout, d'un document pour grand public, la rédaction doit être suffisamment explicite pour être comprise d'un utilisateur occasionnel. 


\subsection{Deuxième étape : modes d'emploi} son concepteur ou à son utilisateur. Il est important que l'étudiant prenne l'habitude de s'informer à la source, quitte à importuner de ses questions le personnel fabricant ou utilisateur. Il convient évidemment de faire comprendre avec tact qu'une telle collaboration apporte une garantie de qualité à la traduction.

- Deuxième constatation : ces erreurs méritent une analyse qui permet de tirer les principes méthodologiques suivants :

- faire les recherches terminologiques en remontant si possible à la source plutôt qu'au dictionnaire (le traducteur japonais a trouvé « cuisine » pour « cooking » dans le dictionnaire et parle donc de la «cuisine de riz » au lieu de la "cuisson du riz », de même " pan » est traduit par « casserole » alors qu'il s'agit d'un « récipient » mais, pour faire ce choix, il faut voir l'appareil) ;

- décider des termes et s'y tenir pour produire un texte homogène (ex : "godet» et « gobelet » alternent pour «cup », « cooking » traduit par « cuisine » est une fois traduit par « cuisson »);

- vérifier l'orthographe de tous les mots. La moindre faute d'orthographe est impardonnable : un mode d'emploi imparfait contribue à dévaloriser le produit ;

- adopter une syntaxe correcte avec des phrases courtes et simples (« au moment où la cuisine de riz prend fin, l'interrupteur est coupé automatiquement et le voyant s'éteint » devient « en fin de cuisson, l'interrupteur se coupe et le voyant s'éteint automatiquement »); 
- vérifier que tous les éléments ont été traduits (une phrase en espagnol s'est subrepticement glissée dans la traduction française) ;

- adopter une présentation claire et lisible (les schémas doivent être explicites : par exemple, les deux modèles doivent être bien différenciés) ;

- valider la traduction en la testant sur l'utilisateur.

\subsubsection{Exemple 2 : le répondeur téléphonique} terminologie et la phraséologie : parlera-t-on de "messages d'accueil », «d'annonces », « de «bandes annonces »? De « lecture » ou " d'écoute » des messages enregistrés ? Va-ton "appuyer" sur une touche, "enclencher» une touche? L'étudiant comprend l'importance de la recherche documentaire, en l'occurrence, la recherche d'autres modes d'emploi rédigés de préférence directement en français ou correctement traduits (attention à ne pas prendre pour référence une mauvaise traduction!).

\subsubsection{Remarques}

47 J'ai insisté sur l'interaction qui doit s'établir entre le traducteur et le donneur d'ordre. Cette interaction est souvent possible puisque, pour les textes opératoires, le donneur d'ordre est soit le fabricant soit l'utilisateur. Puisque nous traduisons vers le français et vivons en France, le client est souvent celui qui commercialise l'appareil, ou alors l'utilisateur qui demande une traduction pour faire fonctionner un produit fabriqué à l'étranger. La collaboration qui s'établit peut-être directement opératoire. Un de nos 
étudiants en DESS, en stage dans une entreprise d'informatique, devait traduire un guide d'utilisation d'un logiciel, initialement rédigé en allemand et en partie traduit - mal vers l'anglais, ce qui lui rendait la tâche très difficile. La validation de la traduction s'est donc faite directement sur la machine. Le traducteur lisait sa traduction, l'ingénieur réalisait les instructions. À chaque blocage, l'ingénieur réfléchissait et disait : « est-ce que ça ne pourrait pas être ça, ou ça? » Le traducteur revenait alors au texte source et en fonction des interférences linguistiques possibles entre l'allemand et l'anglais acquiesçait ou non. L'ingénieur validait alors directement la traduction.

Dans tous les cas, le traducteur ne peut pas se retrancher derrière les erreurs ou les lacunes du texte source. Il doit produire une traduction correcte et opérationnelle, quitte à corriger la rédaction ou la présentation de l'original, quitte à ajouter des informations ou supprimer des évidences pour l'adapter au public.

\subsection{Troisième étape : première approche du texte scientifique}

\subsubsection{Choix du texte}

Il s'agit d'aborder le texte informatif spécialisé à caractère scientifique, mais en choisissant un domaine qui ne soit pas rébarbatif pour les étudiants et, pour commencer, un niveau de langue correspondant aux textes de vulgarisation. Par exemple, la traduction des deux premières pages d'un article tiré de la revue Mechanical Engineering portant sur les différents moyens de lutte contre les marées noires a mis en évidence un certain nombre de difficultés caractéristiques du texte scientifique. Pour ce texte, elles ne portaient pas sur la compréhension, mais sur la réexpression.

\subsubsection{Difficultés d'ordre terminologique}

Terminologie des hydrocarbures avec deux problèmes : d'abord trouver les termes exacts, ensuite régler le problème de la récurrence des termes tels que "oil spill», " oil slick», ainsi que «oil» utilisé comme nom générique ou adjectif et traduit par "pétrole», "hydro-carbures", " pétrolier ", "pétrolifère », etc. Dans un article de ce type, la contrainte stylistique impose une modulation des répétitions qui conduit à jongler avec diverses traductions possibles, en particulier pour « oil spill » traduit par «marée noire », "déversement accidentel de pétrole», "nappe d'hydrocarbures", "catastrophe pétrolière »...

51 Terminologie géographique: si la traduction de «Kuwaiti oil fields» par «champs pétrolifères koweïtiens » s'impose (en prenant garde à l'orthographe), faut-il traduire «Prince William Sound » par la «baie du Prince Guillaume » (tout en respectant les règles typographiques sur l'emploi des majuscules), « Norwuz platform » par la " plate-forme de Nowrouz» (telle qu'elle est citée dans l'Encyclopaedia Universalis)? Ces questions, nouvelles pour les étudiants, méritent une recherche dans les ouvrages de référence, mais la norme est loin d'être évidente. 


\subsubsection{Difficultés de conversion des mesures}

52 Dans cet article américain, les marées noires sont mesurées en gallons, mesure à convertir, mais en quoi ? Les articles français donnent les mesures en tonnes. Il faut donc passer des gallons aux tonnes sans se tromper :

- choisir la bonne mesure de départ (le gallon américain n'est pas le gallon impérial, le premier équivaut à 3,78 , le second à $4,54 \mathrm{l}$ )

- convertir les gallons en litres : 11 millions de gallons x 3,78 = 41,5 millions de litres

- passer des litres aux kilogrammes et pour cela connaître la masse volumique du pétrole (mais quel type de pétrole ?). Le pétrole flotte, donc si 1 litre correspond à peu près à 1 kilogramme, ce qui donne 41,5 millions de kilos

- convertir les kilos en tonnes, soit 41,5 mille tonnes soit 41500 tonnes

Cette opération, banale pour un scientifique utilisant les puissances de 10 au lieu de s'empêtrer dans les mille et les millions, constitue, pour des étudiants en langues, une épreuve que très peu parviennent à franchir. Même si l'étudiant est motivé, le blocage mathématique réapparait au moment de poser l'opération. Dans le groupe de douze étudiants, seulement deux ont réussi à trouver le nombre correct de tonnes. Les erreurs portaient soit sur le choix des unités de mesure soit sur les calculs eux-mêmes.

\subsection{Quatrième étape : l'approche brutale}

\subsubsection{Méthode}

L'étape suivante consiste à mettre l'étudiant au pied du mur en lui assignant la traduction d'un texte technique ou scientifique réellement commandité par un chercheur. Chaque étudiant reçoit un article différent, mais il leur est permis de travailler en binôme s'ils le souhaitent. Ils se trouvent confrontés à un texte long (une quinzaine de pages), inédit, c'est-à-dire à la pointe de la recherche ou de l'innovation (et bien évidemment jamais traduit), dans un domaine qui leur est totalement inconnu. Ils ont un délai à respecter, 4 à 6 semaines, ce qui est d'ailleurs relativement long, mais il faut penser qu'ils suivent d'autres cours et ont d'autres dossiers à faire. Enfin, ils doivent se débrouiller sans l'aide de leur professeur et rendre un travail parfaitement présenté.

Quelques exemples de titres traduits :

GEOMORPHIC EVOLUTION OF THE SAHARA AND THE NILE (géomorphologie)
PUTTING A LID ON NOISE POLLUTION (acoustique)
HAZARD EVALUATION AND WHAT TO DO IF AVALANCHED (nivologie)
THE OBJECT-ORIENTED FUTURE (informatique)
PROFILE-GRINDING MACHINE (mécanique - décolletage)
MILANKOVITCH CYCLES AND THE MEASUREMENT OF TIME (géophysique)

\subsubsection{Bilan de l'expérience}

Les traductions doivent être accompagnées d'un "glossaire de traducteur » que chaque étudiant compose de manière autonome, uniquement en fonction des difficultés inhérentes à l'article, avec l'objectif de pouvoir réutiliser le travail accompli en recherche documentaire et terminologique dans le cas où une autre traduction porterait sur le même sujet. Enfin, chaque étudiant doit présenter au groupe sa méthodologie (analyse des difficultés, description des démarches, justification des choix de traduction) et 
l'essentiel du contenu de l'article. L'expérience est extrêmement convaincante pour les raisons suivantes :

- les étudiants ont été obligés de dominer les sentiments de panique, d'incompréhension et de blocage ressentis à la première lecture du texte. Il leur a fallu pour cela trouver leur propre méthode d'approche du texte : lectures multiples pour appréhender le texte de façon globale et l'apprivoiser, surlignage des passages difficiles, repérage de la terminologie. Ce travail préliminaire leur a appris à cerner le domaine, opération essentielle pour passer à l'étape suivante.

- Ils ont dû effectuer des recherches documentaires pour se familiariser avec le domaine, maîtriser le sujet, comprendre le texte. Parallèlement, ils ont dû faire des recherches terminologiques : dans les deux cas, explorer les bibliothèques, découvrir les centres de documentation spécialisée, se faire accepter et aider par les documentalistes, trouver les dictionnaires adéquats, les manuels et les articles en français aidant à la compréhension du sujet. Cette phase de recherche est indispensable et prend un temps énorme (jusqu'à $80 \%$ du temps consacré à la traduction). Il faut donc être organisé, méthodique, apprendre à gérer son temps et à constituer son propre fonds terminologique et documentaire avec l'outil informatique.

- Il leur a fallu trouver des spécialistes du domaine susceptibles de les éclairer et acceptant de leur consacrer du temps (quitte à négocier un travail de correction d'anglais en échange). Ils ont donc appris à négocier et à gérer des contacts avec des professionnels.

- une fois tout ce travail préparatoire à la compréhension effectué, ils se sont appliqués à rédiger clairement et sans fautes de français ce qu'ils avaient compris, à le saisir sur traitement de textes, et à le mettre en pages

- avant de le soumettre, ils ont dû le tester sur des lecteurs de différentes sortes (lecteur naïf ne connaissant pas le sujet mais jugeant l'expression, lecteur habitué à repérer les fautes de frappe ou d'orthographe, lecteur expert du domaine, etc.)

- Enfin, pour couronner le tout, ils ont communiqué à leurs camarades ce qu'ils avaient compris et traduit.

57 C'est ainsi qu'une étudiante en Maîtrise LEA, titulaire d'un BAC A, qui n'a jamais eu de bonnes notes en mathématiques et en physique, se retrouve en train d'expliquer sereinement et clairement à ses camarades comment assembler les différentes pièces d'une affûteuse à métaux ou comment fonctionne un système anti-bruit...

\section{Conclusions}

\subsection{Un défi didactique}

L'étudiant qui a mené à bien toutes les étapes de cette progression sans se décourager et qui possède une compétence linguistique suffisante dans ses deux langues étrangères me semble apte à poursuivre la formation au niveau supérieur (DESS) où il subit un entraînement intensif à la traduction professionnelle dans les domaines technique et scientifique, mais aussi juridique et économique. Il est confronté à une grande diversité de sujets qui lui permettent de perfectionner la méthode, de travailler plus vite et plus efficacement pour pouvoir affronter le marché.

Le défi n'est cependant relevé que par un petit nombre d'étudiants LEA. Il est vrai que le marché de la traduction ne peut de toute façon absorber des centaines d'étudiants. Ces étudiants LEA qui décident de devenir traducteurs techniques seront-ils vraiment de bons 
traducteurs? Seront-ils plus efficaces que des techniciens ou des scientifiques plus ou moins bilingues qui traduisent eux-mêmes les textes de leur spécialité ?

60 ont réfléchi au processus de traduction et appris une méthode. Ils sont suffisamment motivés et intelligents pour comprendre un sujet complexe, du moins pour atteindre le niveau de compréhension suffisant pour le traduire (qui n'est pas le niveau de compétence du spécialiste). Les difficultés terminologiques sont résolues par le dialogue avec le spécialiste. Néanmoins, il arrive que le traducteur commette des erreurs, faute d'un bagage scientifique suffisant, faute d'une formation de base en sciences.

61 traducteur doit rester vigilant, conscient de ses limites, et être toujours prêt à réviser sa traduction avec un scientifique. Il serait d'ailleurs intéressant de comparer la traduction d'un même article par un scientifique spécialiste et par un traducteur chevronné.

\subsection{Un savoir-faire, des qualités spécifiques}

L'apprentissage de la traduction technique ou spécialisée est long et ardu pour un étudiant de formation LEA et il est vrai que la diversité extrême et le caractère austère des textes à traduire peuvent en rebuter beaucoup. Pourtant, je reprendrai volontiers les termes de Christine Durieux pour revaloriser ce travail: "On doit reconnaître que l'adaptation et la recréation font partie intégrante de la traduction (technique). Traduire, c'est aller vers le lecteur et lui apporter une information qui réponde à son attente " (Durieux 1990 : 178).

Mon expérience d'enseignante me donne à penser que la traduction spécialisée requiert des qualités spécifiques qui sont des qualités personnelles, indépendantes du type de formation initiale. Outre la compétence en deux langues étrangères (et parfois trois ou quatre), et des qualités indispensables de rédacteur en français, ces étudiants-traducteurs se caractérisent par une extraordinaire curiosité, une soif de découvrir et d'apprendre dans les domaines les plus divers. Une étudiante remarquait qu'en abordant la traduction spécialisée, elle avait découvert en elle-même une vocation de détective. Un autre se sent explorateur. Un sujet scientifique austère, une machine compliquée sont pour eux autant de mystères à dominer, de défis à relever. Il y a forcément en eux la passion des langues (ils sont toujours prêts à en apprendre une nouvelle) et surtout celle des mots, ceux de la langue de spécialité qui est considérée comme un code réservé aux initiés dont le traducteur doit percer le secret. L'étudiant-traducteur aime la méthode, l'ordre et le travail bien fait mais sous son apparence de chercheur solitaire et silencieux se cache un véritable enthousiasme pour la connaissance et pour la communication.

\section{BIBLIOGRAPHIE}

Delisle, J. 1980. L'analyse du discours comme méthode de traduction : initiation à la traduction française de textes pragmatiques anglais. Ottawa : Éditions de l'Université d'Ottawa. 
Durieux, C. 1988. Fondement didactique de la traduction technique. Paris : Didier Érudition.

Durieux, C. 1990. « Liberté et créativité en traduction technique ». In Lederer, M \& F. Israel (dir.), La liberté en traduction, Actes du Colloque International. Paris : Didier Érudition.

Gouadec, D. 1989. Le traducteur, la traduction et l'entreprise. Paris : Afnor Gestion.

Grellet, F. 1991. Apprendre à traduire. Typologie d'exercices de traduction. Nancy : Presses

Universitaires de Nancy.

Hurtado Albir, A. 1990. La notion de fidélité en traduction. Paris : Didier Érudition.

Lavault, E. 1985. Fonctions de la traduction en didactique des langues. Paris : Didier Érudition.

Seleskovitch, D. \& Lederer, M. 1984. Interpréter pour traduire. Paris : Didier Érudition.

Vinay, J.P. \& J. Darbelnet. 1988 [1958]. Stylistique comparée du français et de l'anglais. Paris : Didier.

\section{RÉSUMÉS}

Cette communication présente une didactique de la traduction spécialisée adaptée aux étudiants de Langues étrangères appliquées, aux niveaux Licence et Maîtrise, dans le cadre d'une préparation au DESS «Traduction spécialisée et production de textes multilingues" de l'Université Stendhal.

This paper presents a pedagogical approach to specialized translation for third and fourth year students in Applied Foreign Languages, engaged in a postgraduate diploma in "Specialized translation and the production of multilingual texts" at Université Stendhal, Grenoble.

\section{INDEX}

Mots-clés : enseignement, traduction

Keywords : teaching, translation

\section{AUTEUR}

\section{ÉLISABETH LAVAULT-OLLÉON}

Langues Étrangères Appliquées, Université Stendhal Grenoble 3. Elisabeth.Lavault@ugrenoble3.fr 